Proyecciones Journal of Mathematics

Vol. 35, Nº 3, pp. 251-262, September 2016.

Universidad Católica del Norte

Antofagasta - Chile

\title{
Total edge irregularity strength of disjoint union of double wheel graphs
}

\author{
P. Jeyanthi \\ Govindammal Aditanar College for Women, India \\ and \\ A. Sudha \\ Wavoo Wajeeha Women's College of Arts 85 Science, India \\ Received: October 2015. Accepted : March 2016
}

\begin{abstract}
An edge irregular total $k$-labeling $f: V \cup E \rightarrow\{1,2,3, \ldots, k\}$ of a graph $G=(V, E)$ is a labeling of vertices and edges of $G$ in such a way that for any two different edges uv and $u^{\prime} v^{\prime}$ their weights $f(u)+f(u v)+f(v)$ and $f\left(u^{\prime}\right)+f\left(u^{\prime} v^{\prime}\right)+f\left(v^{\prime}\right)$ are distinct. The total edge irregularity strength tes $(G)$ is defined as the minimum $k$ for which the graph $G$ has an edge irregular total k-labeling. In this paper, we determine the total edge irregularity strength of disjoint union of $p$ isomorphic double wheel graphs and disjoint union of $p$ consecutive non-isomorphic double wheel graphs.
\end{abstract}

Keywords: Irregularity strength; total edge irregularity strength; edge irregular total labeling, disjoint union of double wheel graphs.

AMS Classification (2010): 05C78. 


\section{Introduction}

The graphs in this paper are simple, finite and undirected. In [2] Bača et al. defined the notion of edge irregular total $k$-labeling of a graph $G$ as a funtion $\phi: V \cup E \rightarrow\{1,2, \ldots, k\}$ such that the edge weights $w t_{\phi}(u v)=$ $\phi(u)+\phi(u v)+\phi(v)$ are distinct for all the edges. That is $w t_{\phi}(u v) \neq$ $w t_{\phi}\left(u^{\prime} v^{\prime}\right)$ for every pair of edges $u v, u^{\prime} v^{\prime} \in E$. The minimum $k$ for which the graph $G$ has an edge irregular total $k$-labeling is called the total edge irregularity strength of $G$, tes $(G)$. They found a lower bound for the total edge irregularity strength of a graph as

$$
\operatorname{tes}(G) \geq \max \left\{\left\lceil\frac{(|E(G)|+2)}{3}\right\rceil,\left\lceil\frac{(\Delta(G)+1)}{2}\right\rceil\right\}
$$

where $\Delta(G)$ is the maximum degree of $G$. Ivančo and Jendrol̆ [3] posed the following conjecture.

Conjecture:1.1 [3] Let $G$ be an arbitrary graph different from $K_{5}$. Then

$$
\operatorname{tes}(G)=\max \left\{\left\lceil\frac{(|E(G)|+2)}{3}\right\rceil,\left\lceil\frac{(\Delta(G)+1)}{2}\right\rceil\right\}
$$

Conjecture(1.1) has been verified by several authors for several families of graphs. Motivated by the results in $[1,4,5,6]$ we determine the total edge irregularity strength of the disjoint union of double wheel graphs. A double wheel graph $D W_{n}$ of size n can be composed of $2 C_{n}+K_{1}, n \geq 3$, that is it consists of two cycles of size $n$, where all the vertices of the two cycles are connected to a common hub.

\section{Main Results}

In this section, first we determine the total edge irregularity strength of the disjoint union of $p$ isomorphic double wheel graphs with the vertex set $V\left(p D W_{n}\right)=\left\{v_{j}, v_{i}^{j}, u_{i}^{j}: 1 \leq i \leq n, 1 \leq j \leq p\right\}$ and the edge set $E\left(p D W_{n}\right)=\left\{v_{j} v_{i}^{j}, v_{j} u_{i}^{j}, v_{i}^{j} v_{i+1}^{j}, u_{i}^{j} u_{i+1}^{j}\right.$

$1 \leq i \leq n, 1 \leq j \leq p\}$ where the subscript $i$ is taken modulo $n$.

Lemma 2.1. $\operatorname{tes}\left(2 D W_{n}\right)=\left\lceil\frac{8 n+2}{3}\right\rceil, n \geq 3$. 
Proof. Since $\left|E\left(2 D W_{n}\right)\right|=8 n$, tes $\left(2 D W_{n}\right) \geq\left\lceil\frac{8 n+2}{3}\right\rceil$ by (1.1). Let $k=\left\lceil\frac{8 n+2}{3}\right]$. To prove the reverse inequality we define $f: V \cup E \rightarrow$ $\{1,2,3, \ldots, k\}$ as follows:

$f\left(v_{1}\right)=1$

$f\left(v_{2}\right)=n$

$f\left(v_{i}^{1}\right)=i, 1 \leq i \leq n$

$f\left(v_{i}^{2}\right)=k, 1 \leq i \leq n$

$f\left(u_{i}^{1}\right)=n, 1 \leq i \leq n$;

$f\left(u_{i}^{2}\right)=k, 1 \leq i \leq n$

$f\left(v_{1} v_{i}^{1}\right)=1,1 \leq i \leq n$;

$f\left(v_{1} u_{i}^{1}\right)=1+i, 1 \leq i \leq n$

$f\left(v_{i}^{1} v_{i+1}^{1}\right)=2 n+1-i, 1 \leq i \leq n$

$f\left(u_{i}^{1} u_{i+1}^{1}\right)=n+2+i, 1 \leq i \leq n$;

$f\left(v_{2} v_{i}^{2}\right)=3 n+2-k+i, 1 \leq i \leq n$;

$f\left(v_{2} u_{i}^{2}\right)=4 n+2-k+i, 1 \leq i \leq n$;

$f\left(v_{i}^{2} v_{i+1}^{2}\right)=6 n+2-2 k+i, 1 \leq i \leq n$;

$f\left(u_{i}^{2} u_{i+1}^{2}\right)=7 n+2-2 k+i, 1 \leq i \leq n$.

We observe that, $w t\left(v_{1} v_{i}^{1}\right)=2+i, 1 \leq i \leq n$;

$w t\left(v_{1} u_{i}^{1}\right)=n+2+i, 1 \leq i \leq n$;

$w t\left(v_{i}^{1} v_{i+1}^{1}\right)=2 n+2+i, 1 \leq i \leq n$;

$w t\left(u_{i}^{1} u_{i+1}^{1}\right)=3 n+2+i, 1 \leq i \leq n$

$w t\left(v_{2} v_{i}^{2}\right)=4 n+2+i, 1 \leq i \leq n$;

$w t\left(v_{2} u_{i}^{2}\right)=5 n+2+i, 1 \leq i \leq n$;

$w t\left(v_{i}^{2} v_{i+1}^{2}\right)=6 n+2+i, 1 \leq i \leq n ;$

$w t\left(u_{i}^{2} u_{i+1}^{2}\right)=7 n+2+i, 1 \leq i \leq n$.

Theorem 2.2. Let $n \geq 3$ and $p \geq 3$ be two integers. Then the total edge irregularity strength of disjoint union of $p$ isomorphic double wheel graphs is $\left\lceil\frac{4 p n+2}{3}\right\rceil$.

Proof. Since $\left|E\left(p D W_{n}\right)\right|=4 p n$, by (1.1) we have tes $\left(p W_{n}\right) \geq\left\lceil\frac{4 p n+2}{3}\right\rceil$. Let $k=\left\lceil\frac{4 p n+2}{3}\right\rceil$. To prove the reverse inequality, we define the total edge irregular k-labeling $f$ for $1 \leq i \leq n$ and $1 \leq j \leq p$ as follows:

$f\left(v_{j}\right)=f\left(v_{i}^{j}\right)=f\left(u_{i}^{j}\right)=\min \{(j-1) 2 n+1, k\}$.

For $1 \leq j \leq p$ and $(j-1) 2 n+1 \leq k$,

$f\left(v_{j} v_{i}^{j}\right)=i$;

$f\left(v_{j} u_{i}^{j}\right)=n+i$ 
$f\left(v_{i}^{j} v_{i+1}^{j}\right)=2 n+i$

$f\left(u_{i}^{j} u_{i+1}^{j}\right)=3 n+i$.

For $1 \leq j \leq p$ and $(j-1) 2 n+1>k$,

$f\left(v_{j} v_{i}^{j}\right)=4(j-1) n+2-2 k+i$;

$f\left(v_{j} u_{i}^{j}\right)=4(j-1) n+2-2 k+n+i$;

$f\left(v_{i}^{j} v_{i+1}^{j}\right)=4(j-1) n+2-2 k+2 n+i$;

$f\left(u_{i}^{j} u_{i+1}^{j}\right)=4(j-1) n+2-2 k+3 n+i$.

We observe that,

$w t\left(v_{j} v_{i}^{j}\right)=4(j-1) n+2+i, 1 \leq i \leq n, 1 \leq j \leq p$;

$w t\left(v_{j} u_{i}^{j}\right)=4(j-1) n+2+n+i, 1 \leq i \leq n, 1 \leq j \leq p$;

$w t\left(v_{i}^{j} v_{i+1}^{j}\right)=4(j-1) n+2+2 n+i, 1 \leq i \leq n, 1 \leq j \leq p$;

$w t\left(u_{i}^{j} u_{i+1}^{j}\right)=4(j-1) n+2+3 n+i, 1 \leq i \leq n, 1 \leq j \leq p$.

It can be easily verified that all the vertex and edge labels are at most $\mathrm{k}$ and the weights of the edges are pair-wise distinct. Thus the resulting total labeling is the edge irregular total k-labeling. Figure 1 illustrates the edge irregular total labeling of the disjoint union of 4 copies of double wheel graphs. 

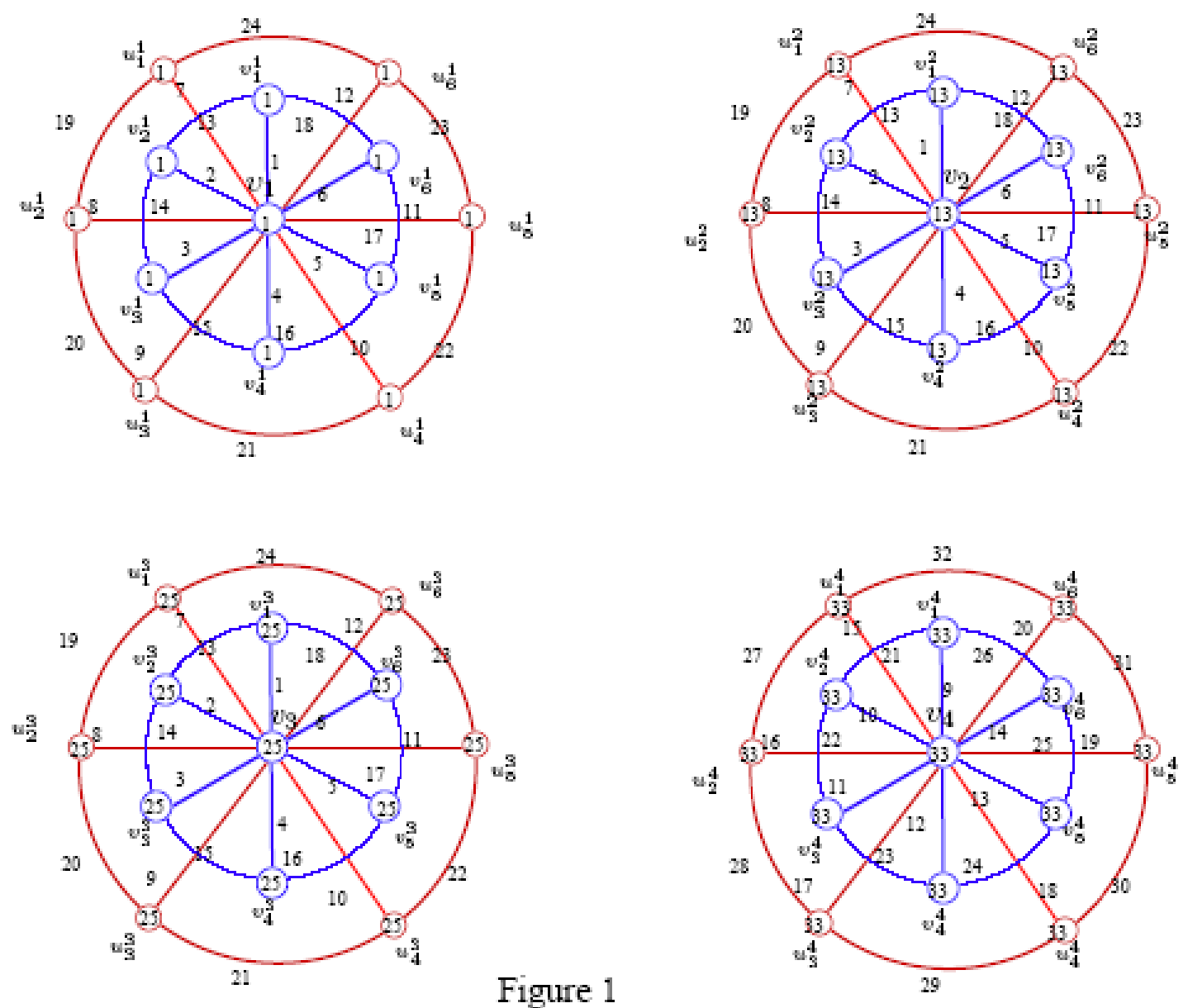

Total edge irregularity strength of disjoint union of 4 isomorphic double Wheel graphs.

$$
\text { tes }\left(4 D W_{6}\right)=33
$$


Now we determine the total edge irregularity strength of the disjoint union of $p$ consecutive $\left(n_{i+1}=n_{i}+1, i \geq 1\right)$ non-isomorphic double wheel graphs with the vertex set $V\left(\bigcup_{j=1}^{p} D W_{n_{j}}\right)$ and the edge set $E\left(\bigcup_{j=1}^{p} D W_{n_{j}}\right)$ where $V\left(\bigcup_{j=1}^{p} D W_{n_{j}}\right)=\left\{v_{j}, v_{i}^{j}, u_{i}^{j}: 1 \leq i \leq n_{j}, 1 \leq j \leq p\right\}$ and $E\left(\bigcup_{j=1}^{p} D W_{n_{j}}\right)=$ $\left\{v_{j} v_{i}^{j}, v_{j} u_{i}^{j}, v_{i}^{j} v_{i+1}^{j}, u_{i}^{j} u_{i+1}^{j}: 1 \leq i \leq n_{j}, 1 \leq j \leq p\right\}$ where the subscript $i$ is taken modulo $n$.

Lemma 2.3. Let $n_{1} \geq 3$ be an integer and $n_{2}=n_{1}+1$. Then $\operatorname{tes}\left(D W_{n_{1}} \cup D W_{n_{2}}\right)=\left\lceil\frac{8 n_{1}+6}{3}\right\rceil$.

Proof. Let $k=\left[\frac{8 n_{1}+6}{3}\right]$. Then by (1.1), tes $\left(D W_{n_{1}} \cup W_{n_{2}}\right) \geq k$. Now to prove the reverse inequality, we define an edge irregular $k$-labeling of $f$ as follows:

$f\left(v_{1}\right)=1$;

$f\left(v_{i}^{1}\right)=i, 1 \leq i \leq n_{1}$

$f\left(v_{i}^{2}\right)=f\left(u_{i}^{2}\right)=k, 1 \leq i \leq n_{2}$;

$f\left(u_{i}^{1}\right)=n_{1}, 1 \leq i \leq n_{1}$

$f\left(v_{2}\right)=n_{1}+1$

$f\left(v_{1} v_{i}^{1}\right)=1,1 \leq i \leq n_{1}$

$f\left(v_{1} u_{i}^{1}\right)=1+i, 1 \leq i \leq n_{1}$;

$f\left(v_{2} v_{i}^{2}\right)=3 n_{1}+1-k+i, 1 \leq i \leq n_{2}$;

$f\left(v_{i}^{1} v_{i+1}^{1}\right)=2 n_{1}+1-i, 1 \leq i \leq n_{1}$;

$f\left(u_{i}^{1} u_{i+1}^{1}\right)=n_{1}+2+i, 1 \leq i \leq n_{1}$;

$f\left(v_{2} u_{i}^{2}\right)=4 n_{1}+2-k+i, 1 \leq i \leq n_{2}$;

$f\left(v_{i}^{2} v_{i+1}^{2}\right)=6 n_{1}+4-2 k+i, 1 \leq i \leq n_{2}$;

$f\left(u_{i}^{2} u_{i+1}^{2}\right)=7 n_{1}+5-2 k+i, 1 \leq i \leq n_{2}$.

We observe that,

for $1 \leq i \leq n_{j}, j=1,2$

$w t\left(v_{1} v_{i}^{1}\right)=2+i$

$w t\left(v_{1} u_{i}^{1}\right)=n_{1}+2+i$

$w t\left(v_{i}^{1} v_{i+1}^{1}\right)=2 n_{1}+2+i$;

$w t\left(u_{i}^{1} u_{i+1}^{1}\right)=3 n_{1}+2+i$;

$w t\left(v_{2} v_{i}^{2}\right)=4 n_{1}+2+i$

$w t\left(v_{2} u_{i}^{2}\right)=5 n_{1}+3+i$

$w t\left(v_{i}^{2} v_{i+1}^{2}\right)=6 n_{1}+4+i$; 
$w t\left(u_{i}^{2} u_{i+1}^{2}\right)=7 n_{1}+5+i$.

It can be easily verified that all the vertex and edge labels are at most $\mathrm{k}$ and the weights of the edges are pair-wise distinct. Thus the resulting total labeling is the edge irregular total k-labeling.

Lemma 2.4. Let $n_{1}, n_{2}, n_{3}$ be integers and $n_{1} \geq 3$. Then tes $\left(D W_{n_{1}} \cup\right.$ $\left.D W_{n_{2}} \cup D W_{n_{3}}\right)=\left\lceil\frac{2\left(6 n_{1}+7\right)}{3}\right\rceil$.

Proof. Since $\left|E\left(D W_{n_{1}} \cup D W_{n_{2}} \cup D W_{n_{3}}\right)\right|=12\left(n_{1}+1\right)$ by (1.1), $\operatorname{tes}\left(D W_{n_{1}} \cup D W_{n_{2}} \cup D W_{n_{3}}\right) \geq\left\lceil\frac{\left.12\left(n_{1}+1\right)+2\right)}{3}\right\rceil=\left\lceil\frac{2\left(6 n_{1}+7\right)}{3}\right\rceil$.Let $k=\left\lceil\frac{2\left(6 n_{1}+7\right)}{3}\right\rceil$.

Now to prove the reverse inequality,we define an edge irregular $k$-labeling $f$ as follows:

$f\left(v_{1}\right)=1$

$f\left(v_{i}^{1}\right)=f\left(u_{i}^{1}\right)=1,1 \leq i \leq n_{1}$;

$f\left(v_{2}\right)=f\left(v_{i}^{2}\right)=f\left(u_{i}^{2}\right)=2 n+1,1 \leq i \leq n_{2}$;

$f\left(v_{3}\right)=3 n_{1}+3$

$f\left(u_{i}^{3}\right)=f\left(v_{i}^{3}\right)=k, 1 \leq i \leq n_{3}$;

$f\left(v_{1} v_{i}^{1}\right)=i, 1 \leq i \leq n_{1}$

$f\left(v_{1} u_{i}^{1}\right)=n_{1}+i, 1 \leq i \leq n_{1}$

$f\left(v_{2} v_{i}^{2}\right)=i, 1 \leq i \leq n_{2}$;

$f\left(v_{2} u_{i}^{2}\right)=n_{1}+1+i, 1 \leq i \leq n_{2} ;$

$f\left(v_{3} v_{i}^{3}\right)=5 n_{1}+3-k+i, 1 \leq i \leq n_{3}$

$f\left(v_{3} u_{i}^{3}\right)=6 n_{1}+5-k+i, 1 \leq i \leq n_{3} ;$

$f\left(v_{i}^{1} v_{i+1}^{1}\right)=2 n_{1}+i, 1 \leq i \leq n_{1}$;

$f\left(u_{i}^{1} u_{i+1}^{1}\right)=3 n_{1}+i, 1 \leq i \leq n_{1}$;

$f\left(v_{i}^{2} v_{i+1}^{2}\right)=2 n_{1}+2+i, 1 \leq i \leq n_{2}$;

$f\left(u_{i}^{2} u_{i+1}^{2}\right)=3 n_{1}+3+i, 1 \leq i \leq n_{2}$;

$f\left(v_{i}^{3} v_{i+1}^{3}\right)=10 n_{1}+10-2 k+i, 1 \leq i \leq n_{3}$;

$f\left(u_{i}^{3} u_{i+1}^{3}\right)=11 n_{1}+12-2 k+i .1 \leq i \leq n_{3}$.

We observe that,

for $1 \leq i \leq n_{j} ; j=1,2,3$

$w t\left(v_{1} v_{i}^{1}\right)=2+i$;

$w t\left(v_{1} u_{i}^{1}\right)=n_{1}+2+i$

$w t\left(v_{2} v_{i}^{2}\right)=4 n_{1}+2+i$

$w t\left(v_{2} u_{i}^{2}\right)=5 n_{1}+3+i$

$w t\left(v_{3} v_{i}^{3}\right)=8 n_{1}+6+i$ 


$$
\begin{aligned}
& w t\left(v_{3} u_{i}^{3}\right)=9 n_{1}+8+i ; \\
& w t\left(v_{i}^{1} v_{i+1}^{1}\right)=2 n_{1}+2+i ; \\
& w t\left(u_{i}^{1} u_{i+1}^{1}\right)=3 n_{1}+2+i \\
& w t\left(v_{i}^{2} v_{i+1}^{2}\right)=6 n_{1}+4+i \\
& w t\left(u_{i}^{2} u_{i+1}^{2}\right)=7 n_{1}+5+i \\
& w t\left(v_{i}^{3} v_{i+1}^{3}\right)=10 n_{1}+10+i \\
& w t\left(u_{i}^{3} u_{i+1}^{3}\right)=11 n_{1}+12+i .
\end{aligned}
$$

It can be easily verified that all the vertex and edge labels are at most $\mathrm{k}$ and the weights of the edges are pair-wise distinct. Thus the resulting total labeling is the edge irregular total k-labeling.

Theorem 2.5. The total edge irregularity strength of the disjoint union of $p(p \geq 4)$ consecutive non-isomorphic double wheel graphs is $\left\lceil\frac{2 p\left(2 n_{1}+p-1\right)+2}{3}\right\rceil$.

Proof. Since $\left|E\left(\bigcup_{j=1}^{p} D W_{n_{j}}\right)\right|=2 p\left(2 n_{1}+p-1\right)$ by (1.1) tes $\left(\bigcup_{j=1}^{p} D W_{n_{j}}\right) \geq$ $\left\lceil\frac{2 p\left(2 n_{1}+p-1\right)+2}{3}\right\rceil$. Let $k=\left\lceil\frac{2 p\left(2 n_{1}+p-1\right)+2}{3}\right\rceil$. To prove the reverse inequality we define the total edge irregular k-labeling $f$ for $1 \leq i \leq n_{j}$ and $1 \leq j \leq p$ as follows:

$$
\begin{aligned}
& f\left(v_{1}\right)=f\left(v_{i}^{1}\right)=f\left(u_{i}^{1}\right)=1,1 \leq i \leq n_{1} ; \\
& f\left(v_{1} v_{i}^{1}\right)=i, 1 \leq i \leq n_{1} ; \\
& f\left(v_{1} u_{i}^{1}\right)=n_{1}+i, 1 \leq i \leq n_{1} ; \\
& f\left(v_{i}^{1} v_{i+1}^{1}\right)=2 n_{1}+i, 1 \leq i \leq n_{1} ; \\
& f\left(u_{i}^{1} u_{i+1}^{1}\right)=3 n_{1}+i, 1 \leq i \leq n_{1} ; \\
& f\left(v_{j}\right)=f\left(v_{i}^{j}\right)=f\left(u_{i}^{j}\right)=\min \left\{2\left(\sum_{s=1}^{j-1} n_{s}\right)+1, k\right\}, 1 \leq i \leq n_{j} \text { and } 2 \leq j \leq \\
& p .
\end{aligned}
$$

For $1 \leq i \leq n_{j}$ and $2 \leq i \leq p, 2\left[\sum_{s=1}^{j-1} n_{s}\right]+1 \leq k$,

$f\left(v_{j} v_{i}^{j}\right)=i$;

$f\left(v_{j} u_{i}^{j}\right)=n_{j}+i$

$f\left(v_{i}^{j} v_{i+1}^{j}\right)=2 n_{j}+i$

$f\left(u_{i}^{j} u_{i+1}^{j}\right)=3 n_{j}+i$.

We observe that,

$\left.w t\left(v_{j} v_{i}^{j}\right)=2\left[2\left(\sum_{s=1}^{j-1} n_{s}\right)\right]+1\right]+i ;$

$w t\left(v_{j} u_{i}^{j}\right)=2\left[2\left(\sum_{s=1}^{j-1} n_{s}\right)+1\right]+n_{j}+i$; 
$w t\left(v_{i}^{j} v_{i+1}^{j}\right)=2\left[2\left(\sum_{s=1}^{j-1} n_{s}\right)+1\right]+2 n_{j}+i ;$

$w t\left(u_{i}^{j} u_{i+1}^{j}\right)=2\left[2\left(\sum_{s=1}^{j-1} n_{s}\right)+1\right]+3 n_{j}+i$.

For $1 \leq i \leq n_{j}$ and $2 \leq i \leq p, 2\left[\sum_{s=1}^{j-1} n_{s}\right]+1>k$,

$f\left(v_{j} v_{i}^{j}\right)=4 n_{j-1}+2\left[2\left(\sum_{s=1}^{j-2} n_{s}\right)+1\right]-2 k+i$

$f\left(v_{j} u_{i}^{j}\right)=4 n_{j-i}+2\left[2\left(\sum_{s=1}^{j-2} n_{s}\right)+1\right]+n_{j}-2 k+i ;$

$f\left(v_{i}^{j} v_{i+1}^{j}\right)=4 n_{j-i}+2\left[2\left(\sum_{s=1}^{j-2} n_{s}\right)+1\right]+2 n_{j}-2 k+i ;$

$f\left(u_{i}^{j} u_{i+1}^{j}\right)=4 n_{j-i}+2\left[2\left(\sum_{s=1}^{j-2} n_{s}\right)+1\right]+3 n_{j}-2 k+i$.

We observe that,

$$
\begin{aligned}
& w t\left(v_{j} v_{i}^{j}\right)=4 n_{j-1}+2\left[2\left(\sum_{s=1}^{j-2} n_{s}\right)+1\right]+i \\
& w t\left(v_{j} u_{i}^{j}\right)=4 n_{j-i}+2\left[2\left(\sum_{s=1}^{j-2} n_{s}\right)+1\right]+n_{j}+i \\
& w t\left(v_{i}^{j} v_{i+1}^{j}\right)=4 n_{j-i}+2\left[2\left(\sum_{s=1}^{j-2} n_{s}\right)+1\right]+2 n_{j}+i \\
& w t\left(u_{i}^{j} u_{i+1}^{j}\right)=4 n_{j-i}+2\left[2\left(\sum_{s=1}^{j-2} n_{s}\right)+1\right]+3 n_{j}+i .
\end{aligned}
$$

It can be easily verified that all the vertex and edge labels are at most $\mathrm{k}$ and the weights of the edges are pair-wise distinct. Thus the resulting total labeling is the edge irregular k-labeling. Figure 2 illustrates the edge irregular total labelings of the disjoint union of 4 Consecutive non-isomorphic double wheel graphs $D W_{3} \cup D W_{4} \cup D W_{5} \cup D W_{6}$. 

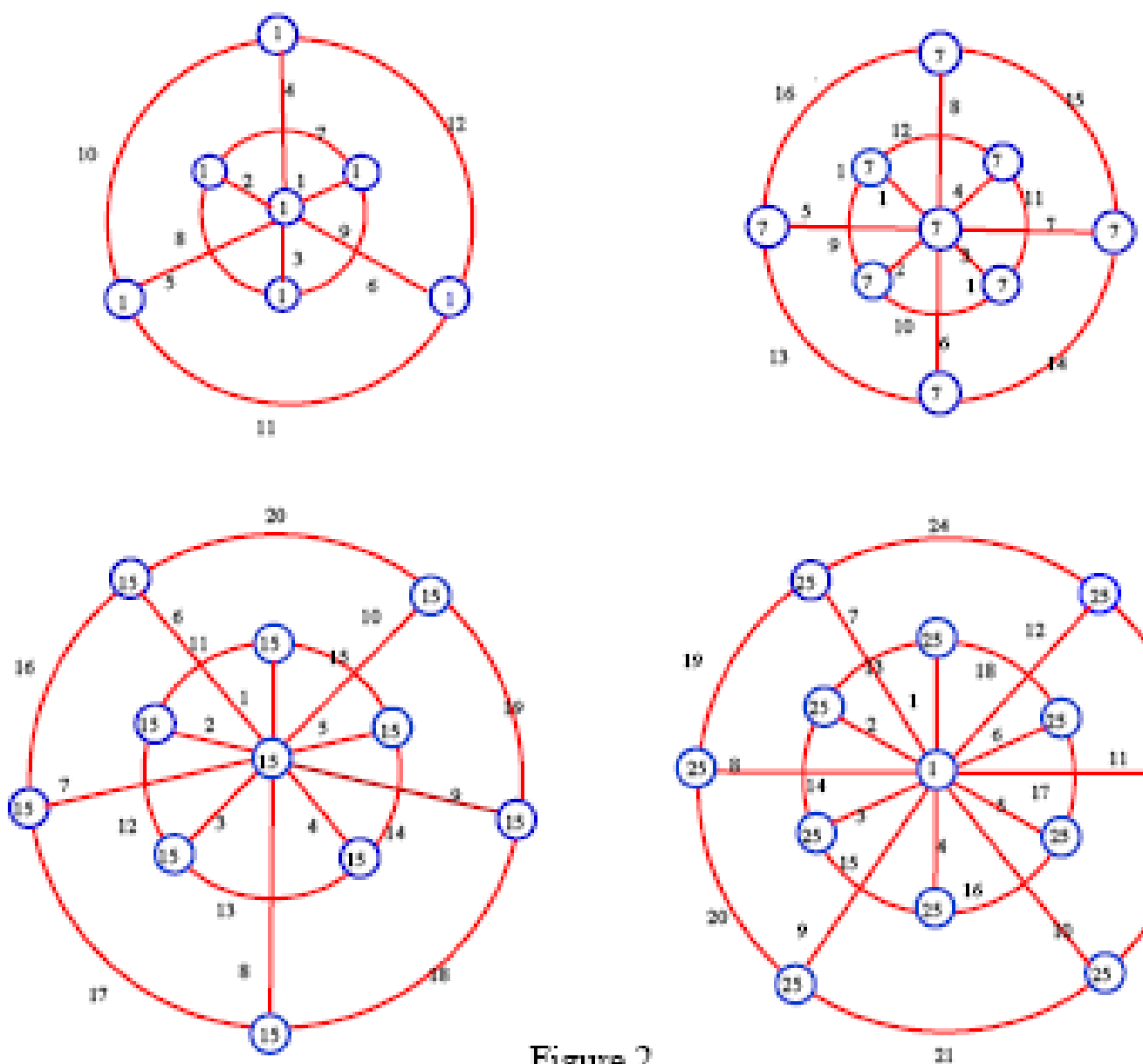

Figure 2

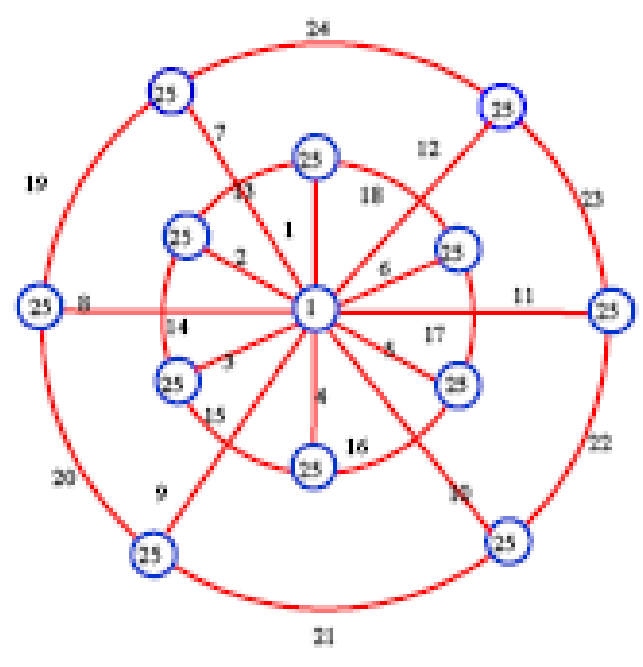

Total edge irregularity strength of disjoint union of 4 non- isomorphic double Wheel graphs.

$$
\operatorname{tes}\left(D W_{3} \bigcup D W_{4} \bigcup D W_{5} \bigcup D W_{6}\right)=25
$$




\section{Conclusion}

In this paper we determine the total edge irregularity strength of the disjoint union of $\mathrm{p}$ isomorphic double wheel graphs and disjoint union of $\mathrm{p}$ consecutive non isomorphic double wheel graphs. We conclude this paper by stating the following open problem.

\section{Open problem:}

For $m \geq 2$,find the exact value of the total edge irregularity strength of a disjoint union of $m$ arbitrary double wheel graphs.

\section{References}

[1] A. Ahmad and M.Bača, and Muhammad Numan, On irregularity strength of the disjoint union of friendship graphs, Electronic Journal of Graph Theory and Applications, 11 (2), pp. 100-108, (2013).

[2] M.Bača,S.Jendrol̆, M. Miller and J. Ryan, On irregular total labellings, Discrete Math., 307, pp. 1378-1388, (2007).

[3] J.Ivančo,S.Jendrol, Total edge irregularity strength of trees, Discussiones Math. Graph Theory, 26, pp. 449-456, (2006).

[4] M.K.Siddiqui,A.Ahmad,M.F.Nadeem,Y.Bashir, Total edge irregularity strength of the disjoint union of sun graphs, International Journal of Mathematics and Soft Computing 3 (1), pp. 21-27, (2013).

[5] P. Jeyanthi and A. Sudha, Total Edge Irregularity Strength of Disjoint Union of Wheel Graphs, Electron. Notes in Discrete Math., 48, pp. 175-182, (2015).

\section{P. Jeyanthi}

Research Centre,

Department of Mathematics

Govindammal Aditanar College for Women

Tiruchendur-628 215, Tamil Nadu,

India

e-mail: jeyajeyanthi@rediffmail.com 
and

\section{A. Sudha}

Department of Mathematics

Wavoo Wajeeha Women's College of Arts \& Science, Kayalpatnam -628 204,Tamil Nadu, India

e-mail: sudhathanalakshmi@gmail.com 